T. Kato and K. Matsumoto

Nagoya Math. J.

Vol. 195 (2009), 113-124

\title{
THE COMMON LIMIT OF A QUADRUPLE SEQUENCE AND THE HYPERGEOMETRIC FUNCTION $\boldsymbol{F}_{D}$ OF THREE VARIABLES
}

\author{
TAKAYUKI KATO AND KEIJI MATSUMOTO
}

\section{Dedicated to Professor Masaaki Yoshida on his sixtieth birthday}

\begin{abstract}
We study a quadruple sequence and express its common limit by Lauricella's hypergeometric function $F_{D}\left(\frac{1}{4}, \frac{1}{4}, \frac{1}{4}, \frac{1}{4}, 1 ; z_{1}, z_{2}, z_{3}\right)$ of three variables. We give a functional equation of $F_{D}$, which is the key to get our expression of the common limit.
\end{abstract}

\section{$\S 1$. Introduction}

For two positive real numbers $a_{0}$ and $b_{0}$ with $a_{0} \geq b_{0}$, the double sequence $\left\{a_{n}\right\}$ and $\left\{b_{n}\right\}$ given as

$$
a_{n+1}=\frac{a_{n}+b_{n}}{2}, \quad b_{n+1}=\sqrt{a_{n} b_{n}}
$$

has a common limit, which is called the arithmetic-geometric mean $M\left(a_{0}, b_{0}\right)$ of $a_{0}$ and $b_{0}$. It is shown by C. F. Gauss that $M\left(a_{0}, b_{0}\right)$ can be expressed by the hypergeometric function:

$$
\frac{a_{0}}{M\left(a_{0}, b_{0}\right)}=\frac{1}{M(1, x)}=F\left(\frac{1}{2}, \frac{1}{2}, 1 ; 1-x^{2}\right),
$$

where $x=b_{0} / a_{0}$.

In this paper, we study a quadruple sequence $\left\{a_{n}\right\},\left\{b_{n}\right\},\left\{c_{n}\right\}$ and $\left\{d_{n}\right\}$

Received August 7, 2007.

Revised June 24, 2008.

Accepted February 2, 2009.

2000 Mathematics Subject Classification: Primary 26A18; Secondary 33C65. 
given by

$$
\begin{array}{cc}
\left(a_{0}, b_{0}, c_{0}, d_{0}\right)=(a, b, c, d), \quad a \geq b \geq c \geq d \geq 0, \\
a_{n+1}=\frac{a_{n}+b_{n}+c_{n}+d_{n}}{4}, \quad b_{n+1}=\frac{\sqrt{\left(a_{n}+d_{n}\right)\left(b_{n}+c_{n}\right)}}{2} \\
c_{n+1}=\frac{\sqrt{\left(a_{n}+c_{n}\right)\left(b_{n}+d_{n}\right)}}{2}, & d_{n+1}=\frac{\sqrt{\left(a_{n}+b_{n}\right)\left(c_{n}+d_{n}\right)}}{2} .
\end{array}
$$

We can easily see that it has a common limit $\mu(a, b, c, d)$. Our main theorem is the expression of $\mu(a, b, c, d)$ by Lauricella's hypergeometric function $F_{D}$ of three variables:

$$
\frac{1}{\mu\left(1, x_{1}, x_{2}, x_{3}\right)}=F_{D}\left(\frac{1}{4}, \frac{1}{4}, \frac{1}{4}, \frac{1}{4}, 1 ; 1-x_{1}^{2}, 1-x_{2}^{2}, 1-x_{3}^{2}\right)^{2} .
$$

The key for our main theorem is Proposition 1, which is the functional equation of the hypergeometric function $F_{D}$ corresponding to the property

$$
\frac{a_{0}}{a_{1}} \mu\left(1, \frac{b_{0}}{a_{0}}, \frac{c_{0}}{a_{0}}, \frac{d_{0}}{a_{0}}\right)=\mu\left(1, \frac{b_{1}}{a_{1}}, \frac{c_{1}}{a_{1}}, \frac{d_{1}}{a_{1}}\right) .
$$

It turns out that

$$
\mu\left(1, \frac{b_{n}}{a_{n}}, \frac{c_{n}}{a_{n}}, \frac{d_{n}}{a_{n}}\right) F_{D}\left(\frac{1}{4}, \frac{1}{4}, \frac{1}{4}, \frac{1}{4}, 1 ; 1-\left(\frac{b_{n}}{a_{n}}\right)^{2}, 1-\left(\frac{c_{n}}{a_{n}}\right)^{2}, 1-\left(\frac{d_{n}}{a_{n}}\right)^{2}\right)^{2}
$$

is independent of $n$. This fact implies our main theorem. In order to show Proposition 1, we prepare some essential facts for integrable Pfaffian systems in Section 3 and give the integrable Pfaffian system with respect to $F_{D}(\alpha, \beta, \gamma ; z)$ of three variables in Fact 4 .

C. W. Borchardt considers in $[B]$ the quadruple sequence

$$
\begin{array}{ll}
a_{n+1}=\frac{a_{n}+b_{n}+c_{n}+d_{n}}{4}, & b_{n+1}=\frac{\sqrt{a_{n} b_{n}}+\sqrt{c_{n} d_{n}}}{2}, \\
c_{n+1}=\frac{\sqrt{a_{n} c_{n}}+\sqrt{b_{n} d_{n}}}{2}, & d_{n+1}=\frac{\sqrt{a_{n} d_{n}}+\sqrt{b_{n} c_{n}}}{2},
\end{array}
$$

with positive initial terms $a_{0}, b_{0}, c_{0}, d_{0}$. Its common limit $B\left(a_{0}, b_{0}, c_{0}, d_{0}\right)$ is expressed in terms of period integrals of a hyperelliptic curve $C$ of genus 2. J. F. Mestre studies the expression of fixed points under the hyperelliptic involution on $C$ by the initial terms and shows that $B\left(a_{0}, b_{0}, c_{0}, d_{0}\right)$ can be expressed by the arithmetic-geometric mean $M\left(a_{0}, c_{0}\right)$ when $a_{0}=b_{0}$ and $c_{0}=d_{0}$, refer to $[\mathrm{M}]$. 
J. M. Borwein and P. B. Borwein consider in $[\mathrm{BB}]$ two double sequences

$$
a_{n+1}=\frac{a_{n}+2 b_{n}}{3}, \quad b_{n+1}=\sqrt[3]{b_{n} \frac{a_{n}^{2}+a_{n} b_{n}+b_{n}^{2}}{3}},
$$

and

$$
a_{n+1}=\frac{a_{n}+3 b_{n}}{4}, \quad b_{n+1}=\sqrt{b_{n} \frac{a_{n}+b_{n}}{2}} ;
$$

they express their common limits $M_{3}\left(a_{0}, b_{0}\right)$ and $M_{4}\left(a_{0}, b_{0}\right)$ by $F\left(\frac{1}{3}, \frac{2}{3}, 1 ; z\right)$ and $F\left(\frac{1}{4}, \frac{3}{4}, 1 ; z\right)$, respectively. We remark that the expression of $M_{4}\left(a_{0}, b_{0}\right)$ can be obtained by our main theorem as a special case $b_{0}=c_{0}=d_{0}$.

As a generalization of $M_{3}\left(a_{0}, b_{0}\right), K$. Koike and H. Shiga give a triple sequence and express its common limit by Appell's hypergeometric function $F_{1}\left(\frac{1}{3}, \frac{1}{3}, \frac{1}{3}, 1 ; z_{1}, z_{2}\right)$ of two variables $z_{1}, z_{2}$, refer to [KS1]. They study an extension of the arithmetic-geometric mean and give its expression by Appell's hypergeometric function $F_{1}$ with different parameters in [KS2].

For other studies related to the arithmetic-geometric mean, refer to $[\mathrm{MM}]$.

Acknowledgment. The authors express their gratitude to Professor K. Ohara for informing of the correct Pfaffian system with respect to the hypergeometric function $F_{D}$ obtained by the system [O].

\section{$\S 2$. The quadruple sequence}

LEMma 1. The quadruple sequence $\left\{a_{n}\right\},\left\{b_{n}\right\},\left\{c_{n}\right\}$ and $\left\{d_{n}\right\}$ given as (1) satisfies

$$
a \geq a_{n-1} \geq a_{n} \geq b_{n} \geq c_{n} \geq d_{n} \geq d_{n-1} \geq d
$$

for any $n \in \mathbb{N}$. It has a common limit, which is denoted by $\mu(a, b, c, d)$.

Proof. We assume $a_{n} \geq b_{n} \geq c_{n} \geq d_{n} \geq 0$ for $n \in \mathbb{N}$. Then we have

$$
\begin{gathered}
\left(4 a_{n+1}\right)^{2}-\left(4 b_{n+1}\right)^{2}=\left(a_{n}-b_{n}-c_{n}+d_{n}\right)^{2} \geq 0 \\
\left(2 b_{n+1}\right)^{2}-\left(2 c_{n+1}\right)^{2}=\left(a_{n}-b_{n}\right)\left(c_{n}-d_{n}\right) \geq 0 \\
\left(2 c_{n+1}\right)^{2}-\left(2 d_{n+1}\right)^{2}=\left(a_{n}-d_{n}\right)\left(b_{n}-c_{n}\right) \geq 0 \\
a_{n+1}=\frac{a_{n}+b_{n}+c_{n}+d_{n}}{4} \leq \frac{a_{n}+a_{n}+a_{n}+a_{n}}{4}=a_{n} \\
d_{n+1}=\frac{\sqrt{\left(a_{n}+b_{n}\right)\left(c_{n}+d_{n}\right)}}{2} \geq \frac{\sqrt{\left(d_{n}+d_{n}\right)\left(d_{n}+d_{n}\right)}}{2}=d_{n}
\end{gathered}
$$


which imply $a_{n} \geq a_{n+1} \geq b_{n+1} \geq c_{n+1} \geq d_{n+1} \geq d_{n} \geq 0$. Since the sequences $\left\{a_{n}\right\}$ and $\left\{d_{n}\right\}$ are monotonous and bounded, they converge. Since

$$
\begin{aligned}
a_{n+1}-d_{n+1} & =\frac{1}{4}\left(\sqrt{a_{n}+b_{n}}-\sqrt{c_{n}+d_{n}}\right)^{2} \leq \frac{1}{4}\left(\sqrt{2 a_{n}}-\sqrt{2 d_{n}}\right)^{2} \\
& =\frac{1}{2}\left(a_{n}+d_{n}-2 \sqrt{a_{n} d_{n}}\right) \leq \frac{a_{n}-d_{n}}{2} \leq \frac{a-d}{2^{n+1}},
\end{aligned}
$$

we have $\lim _{n \rightarrow \infty}\left(a_{n}-d_{n}\right)=0$. Thus the quadruple sequence (1) has a common limit.

Remark 1. 1. We have

$$
\begin{aligned}
& \left(4 a_{n+1}\right)^{2}-\left(4 b_{n+1}\right)^{2}=\left(a_{n}-b_{n}-c_{n}+d_{n}\right)^{2}, \\
& \left(4 a_{n+1}\right)^{2}-\left(4 c_{n+1}\right)^{2}=\left(a_{n}-b_{n}+c_{n}-d_{n}\right)^{2}, \\
& \left(4 a_{n+1}\right)^{2}-\left(4 d_{n+1}\right)^{2}=\left(a_{n}+b_{n}-c_{n}-d_{n}\right)^{2} .
\end{aligned}
$$

2. The quadruple sequence (1) quadratically converges, since

$$
a_{n+1}-d_{n+1} \leq \frac{1}{4}\left(\sqrt{2 a_{n}}-\sqrt{2 d_{n}}\right)^{2}=\frac{1}{2} \frac{\left(a_{n}-d_{n}\right)^{2}}{\left(\sqrt{a_{n}}+\sqrt{d_{n}}\right)^{2}} .
$$

It is easy to see that

$$
\begin{gathered}
\mu(a, b, c, d)=a \mu\left(1, \frac{b}{a}, \frac{c}{a}, \frac{d}{a}\right), \\
\mu(a, b, c, d) \\
=\mu\left(\frac{a+b+c+d}{4}, \frac{\sqrt{(a+d)(b+c)}}{2}, \frac{\sqrt{(a+c)(b+d)}}{2}, \frac{\sqrt{(a+b)(c+d)}}{2}\right) .
\end{gathered}
$$

By putting $x_{1}=b / a, x_{2}=c / a, x_{3}=d / a$ for these equalities, we have the following lemma.

LEMma 2. Let $\left(y_{1}, y_{2}, y_{3}\right)$ be the image of $\left(x_{1}, x_{2}, x_{3}\right)$ by the map $\varphi$

$$
\begin{aligned}
& \varphi\left(x_{1}, x_{2}, x_{3}\right) \\
= & \left(\frac{2 \sqrt{\left(1+x_{3}\right)\left(x_{1}+x_{2}\right)}}{1+x_{1}+x_{2}+x_{3}}, \frac{2 \sqrt{\left(1+x_{2}\right)\left(x_{1}+x_{3}\right)}}{1+x_{1}+x_{2}+x_{3}}, \frac{2 \sqrt{\left(1+x_{1}\right)\left(x_{2}+x_{3}\right)}}{1+x_{1}+x_{2}+x_{3}}\right) .
\end{aligned}
$$

Then $\mu$ satisfies the relation

$$
\frac{4}{1+x_{1}+x_{2}+x_{3}} \mu\left(1, x_{1}, x_{2}, x_{3}\right)=\mu\left(1, y_{1}, y_{2}, y_{3}\right)
$$

for $0<x_{3} \leq x_{2} \leq x_{1} \leq 1$. 


\section{§3. Integrable Pfaffian systems}

In this section, we prepare some facts of integrable Pfaffian systems. We consider a system of first-order partial differential equations with $r$ unknowns $f_{1}, \ldots, f_{r}$ and $n$ variables $x_{1}, \ldots, x_{n}$ in the following form

$$
d f(x)=\Omega(x) f(x),
$$

where $x=\left(x_{1}, \ldots, x_{n}\right)$ is in an open set $U, f(x)={ }^{t}\left(f_{1}(x), \ldots, f_{r}(x)\right)$ and $\Omega(x)$ is an $r \times r$ matrix whose entries are 1 -forms on $U$. The system (3) is called a Pfaffian system on $U$ and $\Omega(x)$ is called the connection matrix of (3). If $\Omega(x)$ satisfies the integrability condition

$$
d \Omega(x)=\Omega(x) \wedge \Omega(x),
$$

then the system (3) is integrable.

FACT 1. 1. The system (3) has exactly $r$ linearly independent vector valued solutions if and only if it is integrable.

2. If the system (3) is integrable, then there exists a unique solution $f$ around $u \in U$ such that $f(u)=p$ for a given initial vector $p \in \mathbb{C}^{r}$.

FACT 2. For an integrable Pfaffian system (3) and an invertible $r \times r$ functional matrix $P(x)$ on $U$, the vector valued function $g(x)=P(x) f(x)$ satisfies the Pfaffian system

$$
d g(x)=\left[P(x) \Omega(x) P(x)^{-1}+d P(x) P(x)^{-1}\right] g(x) .
$$

Let $f_{0}$ be a function of $n$-variables $\left(y_{1}, \ldots, y_{n}\right)$ on an open set $V$. We assume that the vector valued function

$$
f(y)={ }^{t}\left(f_{0}(y), \frac{\partial f_{0}}{\partial y_{1}}(y), \ldots, \frac{\partial f_{0}}{\partial y_{n}}(y)\right)
$$

satisfies an integrable Pfaffian system

$$
d f(y)=\Omega(y) f(y)
$$

on $V$. Let $\eta$ be a map from an open set $U$ to $V$ given as

$$
\eta: U \ni x=\left(x_{1}, \ldots, x_{n}\right) \longmapsto y=\left(\eta_{1}(x), \ldots, \eta_{n}(x)\right) \in V,
$$


and let $J$ be the Jacobi matrix of $\eta$ :

$$
J=\left(\frac{\partial \eta_{i}}{\partial x_{j}}\right)_{i j}=\left(\begin{array}{ccc}
\frac{\partial \eta_{1}}{\partial x_{1}} & \cdots & \frac{\partial \eta_{1}}{\partial x_{n}} \\
\vdots & \cdots & \vdots \\
\frac{\partial \eta_{n}}{\partial x_{1}} & \cdots & \frac{\partial \eta_{n}}{\partial x_{n}}
\end{array}\right)
$$

FACT 3. If $\operatorname{det}(J) \neq 0$ on $U$ then the function $h_{0}(x)=f_{0}(\eta(x))$ satisfies

$$
d h(x)=\left[\tilde{J} \Omega(x) \tilde{J}^{-1}+d \tilde{J} \tilde{J}^{-1}\right] h(x),
$$

where

$$
h(x)={ }^{t}\left(h_{0}(x), \frac{\partial h_{0}}{\partial x_{1}}(x), \ldots, \frac{\partial h_{0}}{\partial x_{n}}(x)\right), \quad \tilde{J}=\left(\begin{array}{cc}
1 & \\
& t_{J}
\end{array}\right),
$$

and $\Omega(x)$ is the pull-back of $\Omega(y)$ under the map $\eta$.

\section{§4. Lauricella's hypergeometric function $\boldsymbol{F}_{\boldsymbol{D}}$}

Lauricella's hypergeometric function $F_{D}$ of $m$-variables $z_{1}, \ldots, z_{m}$ with parameters $\alpha, \beta_{1}, \ldots, \beta_{m}, \gamma$ is defined as

$$
F_{D}(\alpha, \beta, \gamma ; z)=\sum_{n_{1}, \ldots, n_{m} \geq 0}^{\infty} \frac{\left(\alpha, \sum_{j=1}^{m} n_{j}\right) \prod_{j=1}^{m}\left(\beta_{j}, n_{j}\right)}{\left(\gamma, \sum_{j=1}^{m} n_{j}\right) \prod_{j=1}^{m}\left(1, n_{j}\right)} \prod_{j=1}^{m} z_{j}^{n_{j}}
$$

where $z=\left(z_{1}, \ldots, z_{m}\right)$ satisfies $\left|z_{j}\right|<1(j=1, \ldots, m), \beta=\left(\beta_{1}, \ldots, \beta_{m}\right)$, $\gamma \neq 0,-1,-2, \ldots$ and $(\alpha, n)=\alpha(\alpha+1) \cdots(\alpha+n-1)=\Gamma(\alpha+n) / \Gamma(\alpha)$. This function admits the integral representation:

$$
F_{D}(\alpha, \beta, \gamma ; z)=\frac{\Gamma(\gamma)}{\Gamma(\alpha) \Gamma(\gamma-\alpha)} \int_{0}^{1} t^{\alpha}(1-t)^{\gamma-\alpha} \prod_{j=1}^{m}\left(1-z_{j} t\right)^{-\beta_{j}} \frac{d t}{t(1-t)}
$$

When $m=1, F_{D}(\alpha, \beta, \gamma ; z)$ coincides with the Gauss hypergeometric function $F(\alpha, \beta, \gamma ; z)$. We consider $F_{D}\left(\alpha, \beta_{1}, \beta_{2}, \beta_{3}, \gamma ; z_{1}, z_{2}, z_{3}\right)$ of three variables.

FACT 4. The function $F_{D}(\alpha, \beta, \gamma ; z)$ of three variables satisfies the integrable Pfaffian system given as

$$
d f=\sum_{1 \leq i<j \leq 5} A_{i j} d \log \left(z_{i}-z_{j}\right) f
$$


where $f={ }^{t}\left(f_{0}, f_{1}, f_{2}, f_{3}\right), f_{0}=F_{D}(\alpha, \beta, \gamma ; z), f_{i}=z_{i} \frac{\partial f_{0}}{\partial z_{i}}(i=1,2,3)$, $z_{4}=0, z_{5}=1$ and

$$
\begin{aligned}
& A_{12}=\left(\begin{array}{cccc}
0 & 0 & 0 & 0 \\
0 & -\beta_{2} & \beta_{1} & 0 \\
0 & \beta_{2} & -\beta_{1} & 0 \\
0 & 0 & 0 & 0
\end{array}\right), \quad A_{14}=\left(\begin{array}{cccc}
0 & 1 & 0 & 0 \\
0 & 1+\beta_{2}+\beta_{3}-\gamma & 0 & 0 \\
0 & -\beta_{2} & 0 & 0 \\
0 & -\beta_{3} & 0 & 0
\end{array}\right), \\
& A_{13}=\left(\begin{array}{cccc}
0 & 0 & 0 & 0 \\
0 & -\beta_{3} & 0 & \beta_{1} \\
0 & 0 & 0 & 0 \\
0 & \beta_{3} & 0 & -\beta_{1}
\end{array}\right), \quad A_{24}=\left(\begin{array}{cccc}
0 & 0 & 1 & 0 \\
0 & 0 & -\beta_{1} & 0 \\
0 & 0 & 1+\beta_{1}+\beta_{3}-\gamma & 0 \\
0 & 0 & -\beta_{3} & 0
\end{array}\right) \text {, } \\
& A_{23}=\left(\begin{array}{cccc}
0 & 0 & 0 & 0 \\
0 & 0 & 0 & 0 \\
0 & 0 & -\beta_{3} & \beta_{2} \\
0 & 0 & \beta_{3} & -\beta_{2}
\end{array}\right), \quad A_{34}=\left(\begin{array}{cccc}
0 & 0 & 0 & 1 \\
0 & 0 & 0 & -\beta_{1} \\
0 & 0 & 0 & -\beta_{2} \\
0 & 0 & 0 & 1+\beta_{1}+\beta_{2}-\gamma
\end{array}\right) \text {, } \\
& A_{15}=\left(\begin{array}{cccc}
0 & 0 & 0 & 0 \\
-\alpha \beta_{1} & \gamma-\alpha-\beta_{1}-1 & -\beta_{1} & -\beta_{1} \\
0 & 0 & 0 & 0 \\
0 & 0 & 0 & 0
\end{array}\right) \text {, } \\
& A_{25}=\left(\begin{array}{cccc}
0 & 0 & 0 & 0 \\
0 & 0 & 0 & 0 \\
-\alpha \beta_{2} & -\beta_{2} & \gamma-\alpha-\beta_{2}-1 & -\beta_{2} \\
0 & 0 & 0 & 0
\end{array}\right) \text {, } \\
& A_{35}=\left(\begin{array}{cccc}
0 & 0 & 0 & 0 \\
0 & 0 & 0 & 0 \\
0 & 0 & 0 & 0 \\
-\alpha \beta_{3} & -\beta_{3} & -\beta_{3} & \gamma-\alpha-\beta_{3}-1
\end{array}\right) \text {. }
\end{aligned}
$$

Remark 2. The $A_{i j}$ and $A_{i, n+1}$ in the proof of Proposition 9.1.4 in [IKSY] are wrong. Professor K. Ohara informed us of the correct Pfaffian system given by the system $[\mathrm{O}]$.

\section{$\S 5$. Main theorem}

THEOREM 1. For any numbers $x_{1}, x_{2}, x_{3}$ satisfying $0<x_{3} \leq x_{2} \leq$ $x_{1} \leq 1$, we have

$$
\frac{1}{\mu\left(1, x_{1}, x_{2}, x_{3}\right)}=F_{D}\left(\frac{1}{4}, \frac{1}{4}, \frac{1}{4}, \frac{1}{4}, 1 ; 1-x_{1}^{2}, 1-x_{2}^{2}, 1-x_{3}^{2}\right)^{2},
$$


where $\mu\left(1, x_{1}, x_{2}, x_{3}\right)$ is the common limit of the quadruple sequence (1) with initial $\left(1, x_{1}, x_{2}, x_{3}\right)$ and $F_{D}$ is Lauricella's hypergeometric function.

We put

$$
F\left(z_{1}, z_{2}, z_{3}\right)=F_{D}\left(\frac{1}{4}, \frac{1}{4}, \frac{1}{4}, \frac{1}{4}, 1 ; z_{1}, z_{2}, z_{3}\right) .
$$

Proposition 1. The function $F$ satisfies

$$
\begin{aligned}
& \frac{1+x_{1}+x_{2}+x_{3}}{4} F\left(1-x_{1}^{2}, 1-x_{2}^{2}, 1-x_{3}^{2}\right)^{2}=F\left(1-y_{1}^{2}, 1-y_{2}^{2}, 1-y_{3}^{2}\right)^{2} \\
= & F\left(\left(\frac{1-x_{1}-x_{2}+x_{3}}{1+x_{1}+x_{2}+x_{3}}\right)^{2},\left(\frac{1-x_{1}+x_{2}-x_{3}}{1+x_{1}+x_{2}+x_{3}}\right)^{2},\left(\frac{1+x_{1}-x_{2}-x_{3}}{1+x_{1}+x_{2}+x_{3}}\right)^{2}\right)^{2},
\end{aligned}
$$

where $\left(y_{1}, y_{2}, y_{3}\right)=\varphi\left(x_{1}, x_{2}, x_{3}\right)$ is defined in Lemma 2 .

Proof. Put

$$
\left(\xi_{1}, \xi_{2}, \xi_{3}\right)=\left(\frac{1-x_{1}-x_{2}+x_{3}}{1+x_{1}+x_{2}+x_{3}}, \frac{1-x_{1}+x_{2}-x_{3}}{1+x_{1}+x_{2}+x_{3}}, \frac{1+x_{1}-x_{2}-x_{3}}{1+x_{1}+x_{2}+x_{3}}\right) .
$$

Then we have

$$
\begin{aligned}
& \left(x_{1}, x_{2}, x_{3}\right)=\left(\frac{1-\xi_{1}-\xi_{2}+\xi_{3}}{1+\xi_{1}+\xi_{2}+\xi_{3}}, \frac{1-\xi_{1}+\xi_{2}-\xi_{3}}{1+\xi_{1}+\xi_{2}+\xi_{3}}, \frac{1+\xi_{1}-\xi_{2}-\xi_{3}}{1+\xi_{1}+\xi_{2}+\xi_{3}}\right), \\
& \frac{1+x_{1}+x_{2}+x_{3}}{4}=\frac{1}{1+\xi_{1}+\xi_{2}+\xi_{3}}, \\
& \left(1-x_{1}^{2}, 1-x_{2}^{2}, 1-x_{3}^{2}\right) \\
= & \left(\frac{4\left(1+\xi_{3}\right)\left(\xi_{1}+\xi_{2}\right)}{\left(1+\xi_{1}+\xi_{2}+\xi_{3}\right)^{2}}, \frac{4\left(1+\xi_{2}\right)\left(\xi_{1}+\xi_{3}\right)}{\left(1+\xi_{1}+\xi_{2}+\xi_{3}\right)^{2}}, \frac{4\left(1+\xi_{1}\right)\left(\xi_{2}+\xi_{3}\right)}{\left(1+\xi_{1}+\xi_{2}+\xi_{3}\right)^{2}}\right) .
\end{aligned}
$$

Thus the equality in Proposition 1 is equivalent to

$$
\begin{aligned}
& \sqrt{1+\xi_{1}+\xi_{2}+\xi_{3}} F\left(\xi_{1}^{2}, \xi_{2}^{2}, \xi_{3}^{2}\right) \\
= & F\left(\frac{4\left(1+\xi_{3}\right)\left(\xi_{1}+\xi_{2}\right)}{\left(1+\xi_{1}+\xi_{2}+\xi_{3}\right)^{2}}, \frac{4\left(1+\xi_{2}\right)\left(\xi_{1}+\xi_{3}\right)}{\left(1+\xi_{1}+\xi_{2}+\xi_{3}\right)^{2}}, \frac{4\left(1+\xi_{1}\right)\left(\xi_{2}+\xi_{3}\right)}{\left(1+\xi_{1}+\xi_{2}+\xi_{3}\right)^{2}}\right)
\end{aligned}
$$

for $0 \leq \xi_{1} \leq \xi_{2} \leq \xi_{3}<1$. We show that the Pfaffian systems obtained by the functions in the both sides of the above equality coincide.

Let $\Omega(x)$ be the connection 1-form in Fact 4 for $\alpha=\beta_{1}=\beta_{2}=\beta_{3}=1 / 4$ and $\gamma=1$. Fact 2 implies that the vector valued function

$$
g(x)={ }^{t}\left(F, \frac{\partial F}{\partial x_{1}}, \frac{\partial F}{\partial x_{2}}, \frac{\partial F}{\partial x_{3}}\right)
$$


satisfies the Pfaffian system $d g=\Omega_{1}(x) g$, where

$$
\begin{aligned}
& \Omega_{1}(x)=P \Omega(x) P^{-1}+d P P^{-1}, \\
& P=\operatorname{diag}\left(1, \frac{1}{x_{1}}, \frac{1}{x_{2}}, \frac{1}{x_{3}}\right)=\left(\begin{array}{llll}
1 & & & \\
& \frac{1}{x_{1}} & & \\
& & \frac{1}{x_{2}} & \\
& & & \frac{1}{x_{3}}
\end{array}\right) .
\end{aligned}
$$

The vector valued function

$$
h(\xi)={ }^{t}\left(h_{0}, \frac{\partial h_{0}}{\partial \xi_{1}}, \frac{\partial h_{0}}{\partial \xi_{2}}, \frac{\partial h_{0}}{\partial \xi_{3}}\right)
$$

for

$$
h_{0}\left(\xi_{1}, \xi_{2}, \xi_{3}\right)=\sqrt{1+\xi_{1}+\xi_{2}+\xi_{3}} F\left(\xi_{1}^{2}, \xi_{2}^{2}, \xi_{3}^{2}\right)
$$

satisfies $h(0,0,0)={ }^{t}(1,1 / 2,1 / 2,1 / 2)$ and the Pfaffian system $d h=\Omega_{2}(\xi) h$, where

$$
\begin{aligned}
& \Omega_{2}(\xi)=Q\left[J_{1} \Omega_{1}(\xi) J_{1}^{-1}+d J_{1} J_{1}^{-1}\right] Q^{-1}+d Q Q^{-1}, \quad J_{1}=\operatorname{diag}\left(1,2 \xi_{1}, 2 \xi_{2}, 2 \xi_{3}\right),
\end{aligned}
$$

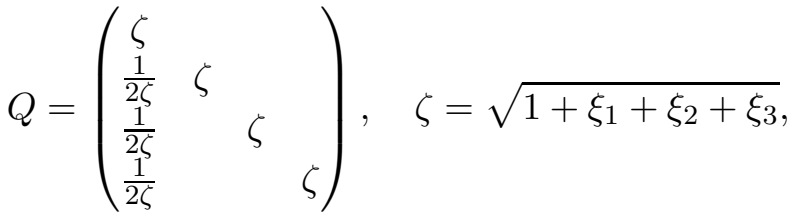

and $\Omega_{1}(\xi)$ is the pull-back of $\Omega_{1}(x)$ under the map

$$
\left(\xi_{1}, \xi_{2}, \xi_{3}\right) \longmapsto\left(x_{1}, x_{2}, x_{3}\right)=\left(\xi_{1}^{2}, \xi_{2}^{2}, \xi_{3}^{2}\right) .
$$

On the other hand, the vector valued function

$$
h(x)=t\left(h_{0}, \frac{\partial h_{0}}{\partial \xi_{1}}, \frac{\partial h_{0}}{\partial \xi_{2}}, \frac{\partial h_{0}}{\partial \xi_{3}}\right)
$$

for

$$
h_{0}\left(\xi_{1}, \xi_{2}, \xi_{3}\right)=F\left(\frac{4\left(1+\xi_{3}\right)\left(\xi_{1}+\xi_{2}\right)}{\left(1+\xi_{1}+\xi_{2}+\xi_{3}\right)^{2}}, \frac{4\left(1+\xi_{2}\right)\left(\xi_{1}+\xi_{3}\right)}{\left(1+\xi_{1}+\xi_{2}+\xi_{3}\right)^{2}}, \frac{4\left(1+\xi_{1}\right)\left(\xi_{2}+\xi_{3}\right)}{\left(1+\xi_{1}+\xi_{2}+\xi_{3}\right)^{2}}\right)
$$

satisfies $h(0,0,0)={ }^{t}(1,1 / 2,1 / 2,1 / 2)$ and the Pfaffian system $d h=\Omega_{3}(\xi) h$, where

$$
\Omega_{3}(\xi)=J_{2} \Omega_{1}^{\prime}(\xi) J_{2}^{-1}+d J_{2} J_{2}^{-1}, \quad J_{2}=\left(\begin{array}{ll}
1 & \\
& t_{J}
\end{array}\right),
$$


$\Omega_{1}^{\prime}(\xi)$ is the pull-back of $\Omega_{1}(x)$ under the map

$$
\varphi^{\prime}:\left(\xi_{1}, \xi_{2}, \xi_{3}\right) \mapsto\left(\frac{4\left(1+\xi_{3}\right)\left(\xi_{1}+\xi_{2}\right)}{\left(1+\xi_{1}+\xi_{2}+\xi_{3}\right)^{2}}, \frac{4\left(1+\xi_{2}\right)\left(\xi_{1}+\xi_{3}\right)}{\left(1+\xi_{1}+\xi_{2}+\xi_{3}\right)^{2}}, \frac{4\left(1+\xi_{1}\right)\left(\xi_{2}+\xi_{3}\right)}{\left(1+\xi_{1}+\xi_{2}+\xi_{3}\right)^{2}}\right),
$$

and $J$ is the Jacobi matrix of the map $\varphi^{\prime}$. By a straight forward calculation, we can show that $\Omega_{2}(\xi)=\Omega_{3}(\xi)$. Thus we have the required equality around $\xi=(0,0,0)$.

Proof of Theorem 1. Consider the quadruple sequence (1) with initial $\left(a_{0}, b_{0}, c_{0}, d_{0}\right)=\left(1, x_{1}, x_{2}, x_{3}\right)$. Lemma 2 and Proposition 1 imply that

$$
\begin{aligned}
& \mu\left(1, x_{1}, x_{2}, x_{3}\right) F\left(1-x_{1}^{2}, 1-x_{2}^{2}, 1-x_{3}^{2}\right)^{2} \\
= & \mu\left(1, y_{1}, y_{2}, y_{3}\right) F\left(1-y_{1}^{2}, 1-y_{2}^{2}, 1-y_{3}^{2}\right)^{2} .
\end{aligned}
$$

Thus we have

$$
\begin{aligned}
& \mu\left(1, x_{1}, x_{2}, x_{3}\right) F\left(1-x_{1}^{2}, 1-x_{2}^{2}, 1-x_{3}^{2}\right)^{2} \\
= & \mu\left(1, \frac{b_{n}}{a_{n}}, \frac{c_{n}}{a_{n}}, \frac{d_{n}}{a_{n}}\right) F\left(1-\left(\frac{b_{n}}{a_{n}}\right)^{2}, 1-\left(\frac{c_{n}}{a_{n}}\right)^{2}, 1-\left(\frac{d_{n}}{a_{n}}\right)^{2}\right)^{2}
\end{aligned}
$$

for any $n \in \mathbb{N}$. Since

$$
\lim _{n \rightarrow \infty} \frac{b_{n}}{a_{n}}=\lim _{n \rightarrow \infty} \frac{c_{n}}{a_{n}}=\lim _{n \rightarrow \infty} \frac{d_{n}}{a_{n}}=1,
$$

and $\mu(1,1,1,1)=F(0,0,0)=1$, we have

$$
\begin{aligned}
& \mu\left(1, x_{1}, x_{2}, x_{3}\right) F\left(1-x_{1}^{2}, 1-x_{2}^{2}, 1-x_{3}^{2}\right)^{2} \\
= & \lim _{n \rightarrow \infty} \mu\left(1, \frac{b_{n}}{a_{n}}, \frac{c_{n}}{a_{n}}, \frac{d_{n}}{a_{n}}\right) F\left(1-\left(\frac{b_{n}}{a_{n}}\right)^{2}, 1-\left(\frac{c_{n}}{a_{n}}\right)^{2}, 1-\left(\frac{d_{n}}{a_{n}}\right)^{2}\right)^{2} \\
= & \mu(1,1,1,1) F(0,0,0)^{2}=1,
\end{aligned}
$$

which is the desired equality.

Corollary 1. For $1>x_{1} \geq x_{2} \geq x_{3} \geq 0$, we have

$$
F\left(1-x_{1}^{2}, 1-x_{2}^{2}, 1-x_{3}^{2}\right)=\prod_{n=0}^{\infty} \sqrt{\frac{a_{n}}{a_{n+1}}},
$$

where we set the initial of the quadruple sequence (1) as $\left(a_{0}, b_{0}, c_{0}, d_{0}\right)=$ $\left(1, x_{1}, x_{2}, x_{3}\right)$. 
Proof. By Proposition 1, we have

$$
\begin{aligned}
& F\left(1-x_{1}^{2}, 1-x_{2}^{2}, 1-x_{3}^{2}\right) \\
= & \frac{2}{\sqrt{1+x_{1}+x_{2}+x_{3}}} F\left(1-y_{1}^{2}, 1-y_{2}^{2}, 1-y_{3}^{2}\right) \\
= & \sqrt{\frac{4 a_{0}}{a_{0}+b_{0}+c_{0}+d_{0}}} F\left(1-\frac{b_{1}^{2}}{a_{1}^{2}}, 1-\frac{c_{1}^{2}}{a_{1}^{2}}, 1-\frac{d_{1}^{2}}{a_{1}^{2}}\right) \\
= & \sqrt{\frac{a_{0}}{a_{1}}} \sqrt{\frac{a_{1}}{a_{2}}} F\left(1-\frac{b_{2}^{2}}{a_{2}^{2}}, 1-\frac{c_{2}^{2}}{a_{2}^{2}}, 1-\frac{d_{2}^{2}}{a_{2}^{2}}\right) \\
= & \left(\prod_{i=0}^{n-1} \sqrt{\frac{a_{i}}{a_{i+1}}}\right) F\left(1-\frac{b_{n}^{2}}{a_{n}^{2}}, 1-\frac{c_{n}^{2}}{a_{n}^{2}}, 1-\frac{d_{n}^{2}}{a_{n}^{2}}\right),
\end{aligned}
$$

which implies this corollary.

\section{§6. A specialization}

For the case $b=c=d$, the quadruple sequence reduces to

$$
a_{n+1}=\frac{a_{n}+3 b_{n}}{4}, \quad b_{n+1}=c_{n+1}=d_{n+1}=\sqrt{b_{n} \frac{a_{n}+b_{n}}{2}},
$$

which is studied in $[\mathrm{BB}]$. It is shown that the reciprocal of the common limit of the double sequences is $F\left(\frac{1}{4}, \frac{3}{4}, 1 ; 1-x^{2}\right)^{2}$, where $x=b / a$ and $F(\alpha, \beta, \gamma ; z)$ is the Gauss hypergeometric function. By our main theorem, we have

$$
\begin{aligned}
& F_{D}\left(\frac{1}{4}, \frac{1}{4}, \frac{1}{4}, \frac{1}{4}, 1 ; 1-x^{2}, 1-x^{2}, 1-x^{2}\right)^{2} \\
= & \frac{1}{\mu(1, x, x, x)}=\frac{1}{M_{4}(1, x)}=F\left(\frac{1}{4}, \frac{3}{4}, 1 ; 1-x^{2}\right)^{2} .
\end{aligned}
$$

Note that the above reduction of $F_{D}$ to $F$ can be easily obtained by the integral representation of $F_{D}$.

\section{REFERENCES}

[B] C. W. Borchardt, Über das arithmetisch-geometrische Mittel aus vier Elementen, Berl. Monatsber, 53 (1876), 611-621.

[BB] J. M. Borwein and P. B. Borwein, A cubic counterpart of Jacobi's identity and the AGM, Trans. Amer. Math. Soc., 323 (2) (1991), 691-701.

[IKSY] K. Iwasaki, H. Kimura, S. Shimomura and M. Yoshida, From Gauss to Painlevé, Vieweg, Braunschweig, Wiesbaden, 1991. 
[KS1] K. Koike and H. Shiga, Isogeny formulas for the Picard modular form and a three terms arithmetic geometric mean, J. Number Theory, 124 (2007), 123-141.

[KS2] K. Koike and H. Shiga, Extended Gauss AGM and corresponding Picard modular forms, J. Number Theory, 128 (2008), 2097-2126.

[M] J. F. Mestre, Moyenne de Borchardt et intégrales elliptiques, C. R. Acad. Sci. Paris, 313 (1991), 273-276.

[MM] D. V. Manna and V. H. Moll, Landen survey, Probability, Geometry and Integrable Systems, MSRI Publications, 55, Cambridge University Press, Cambridge, 2008, pp. 287-319.

[O] K. Ohara, yang - a package for computation in the ring of differential-difference operators, http://www.openxm.org, 2007.

Takayuki Kato

$M E C I N C$.

2-6 Futuka, Aoba-ku

Sendai 980-0802

Japan

katou-t@mec-inc.co.jp

Keiji Matsumoto

Department of Mathematics

Hokkaido University

Sapporo 060-0810

Japan

matsu@math.sci.hokudai.ac.jp 